32) Rowe, P. W., B. A. Partridge and E. Lyall: ibid., 19, 973 (1964)

33) Rowe, P.N. and B.A. Partridge: Trans. Inst. Chem. Engrs., 43, 157 (1965)

34) Squires, A. M.: Chem. Eng. Prog., 58, No. 4, 66 (1962)

35) Shen, C. Y. and H. F. Johnston: AIChE J., 1, 349 (1955)
36) Toei, R., R. Matsuno, H. Kojima, K. Nakagawa and S. Yu: Kagaku Kōgaku, 29, 851 (1965)

37) Van Deemter, J. J.: Chem. Eng. Sci., 13, 143 (1961)

38) Van Deemter, J. J.: Intern. Symp. on Fluidization, Eindhoven, p. 334 (1967)

\title{
BEHAVIOUR OF SUCCESSIVE CONTACT MODEL FOR CATALYTIC REACTION IN FLUID BEDS*
}

\author{
TERUKATSU MIYAUCHI \\ Department of Chemical Engineering, University of Tokyo, Tokyo
}

\begin{abstract}
In a companion paper (J. Chem. Eng. Japan, 7, 201, 1974), a concept of the successive contact model has been presented to account for the extent of catalytic reaction in a fluid bed. The model has been tested here for its soundness by comparing the behaviour of model with catalytic reaction data available so far.

An apparent fraction of catalyst $\mu^{*}$ in the bubble phase for catalytic hydrogenation of ethylene is shown to be reasonably well estimated from catalyst distribution in the dilute phase. The model provides a sound explanation for an apparent anomaly of $\mu^{*}$ being fairly greater than unity in some data. Influence of bed design and operation on selectivity of a given catalytic reaction is briefly given for fluid beds.
\end{abstract}

\section{Introduction}

In a companion paper $^{14)}$ a concept of successive contact model has been introduced to take into consideration the contribution of dilute phase catalyst to overall conversion of catalytic reaction. The reason of introducing this model was primarily to avoid the difficulty observed by Gilliland and Knudsen ${ }^{7)}$ for usual two phase models of fluid beds.

In what follows, the model will be tested for its soundness by comparing its performance with experimental data available so far. Those given by Lewis $^{12)}$ and Gilliland ${ }^{7}$ are extensively utilized here, since their reaction data are taken under relatively higher rate of reaction and provide the severest test of models. It is usually hard to distinguish the soundness of one model from another when reaction rate is slow or moderate, since the reaction taking place in the emulsion phase bulk becomes controlling for the progress of overall conversion.

\section{Preliminary Consideration}

The reaction used by Lewis $^{12)}$ was hydrogenation of ethylene catalysed by a nickel-coated cracking catalyst at an average temperature of $113^{\circ} \mathrm{C}$ under about atmospheric pressure. Reaction occurred with

* Received on August 24, 1973

干113 東京都文京区本郷7-3-1

東京大学工学部化学工学科 宮内照勝 a large excess of ethylene present, so that the reaction was first-order in hydrogen and the reverse reaction was negligible. The particles fluidized consisted primarily of the $100 \sim 140$ mesh fraction (average particle diameter $d_{p}=122 \mu$ ) of microspherical Aerocat (MS-A) cracking catalyst. Quiescent bed density was $0.52 \mathrm{~g} / \mathrm{cc}$ and quiescent gas velocity was $0.73 \mathrm{~cm} / \mathrm{sec}$. Some work was also done with two fractions of impregnated alumina of 188 and $80 \mu$ in $d_{p}$.

Their fluidized bed consisted of a $4 \mathrm{ft}$. length of 2.05 in. i.d. Pyrex tubing. Physical parameters they used were mainly those based on the quiescent bed density. According to Morooka et al. ${ }^{18)}$, this bed density is about 1.09 times greater than the emulsion phase density during fluidization for a fluid bed of FCC catalyst (which has the quiescent bed density of $0.54 \mathrm{~g} / \mathrm{cc})$. The factor $\lambda(=1.09$ in the above case) is found ${ }^{18)}$ to be rather independent of gas velocity and bed diameter. Lewis determined catalyst activity $K$ by a fixed-bed catalyst tester, and correlated their fluid bed data according to the direct contact model of the VUE modefication. Based on these, they obtained the fraction of catalyst in the bubble phase, $a$, and the gas exchange rate, $F$.

Physical quantities which correspond to $K, a$, and $F$ are $k_{r}, \mu^{*}$ and $k_{o b} a_{b}$, respectively. Relationships between these variables are given as follows:

$$
K=\lambda k_{r}
$$




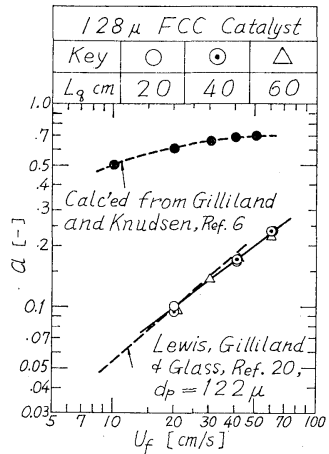

(a)

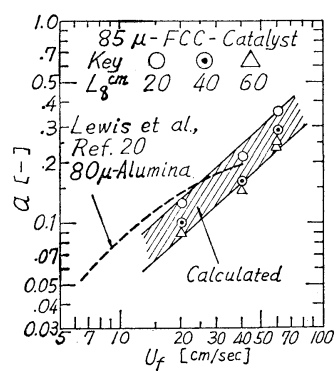

(b)
Fig. 1 Comparison of experimental " $a$ " with calculation based on successive contact model

$a=$ total volume of catalyst in bubble phase $a=\frac{\text { total volume of catalyst in whole bed }}{\mu^{*} \varepsilon_{b}}$

$$
=\frac{\mu^{*} \varepsilon_{b}}{\varepsilon_{e}+\mu^{*} \varepsilon_{b}}
$$

$F=$ volumetric rate of gas exchange

$F=$ volume of quiescent emulsion phase

$$
=\lambda\left(\frac{k_{o b} a_{b}}{\varepsilon_{e}}\right)
$$

To see the applicability of the VUE modification to the data by Lewis, the range of $\varphi$ and $\xi$ (ref. to Eqs. (15) and (13) of ref. 14) have been estimated in what follows. The partition ratio $m$ of hydrogen was not measured. However, $m$ for FCC catalyst (without nickel impregnation) at room temperature and atmospheric pressure was found as 0.83 when measured by a chromatographic method. Allowing slight adsorption of hydrogen on nickel, $m$ has been taken as unity under the reaction conditions.

Possible approximate numerical values are estimated from the literature for such variables as $k_{b}{ }^{16,23)}$, $a_{b}{ }^{17)}, \varepsilon_{e}\left(=1-\varepsilon_{b}\right)^{18)}, k_{e x} a_{e x}{ }^{11,16)}$, and $k_{r}{ }^{12)}$.

The overall coefficient of mass transfer $k_{o b}$ has been calculated from Eq. (A.6) by assuming freely rising bubbles (ref. to Appendix). When the bubbles are retarded by the wall effect, $\varphi$ values are still higher than those for the freely rising bubbles.

The order of magnitude of variables estimated for operational range of $U_{f}=10 \sim 40 \mathrm{~cm} / \mathrm{sec}$ is as follows; $k_{o b} a_{b} \approx 0.35 \mathrm{sec}^{-1}, \quad \varepsilon_{e u} u_{e u} / U_{f} \approx 1, \quad k_{e x} a_{e x} \approx 4 \sim 10 \mathrm{sec}^{-1}$, $\beta_{r} \approx 1 \sim 1.5$ and $\varepsilon_{e} k_{r} \approx 1 \sim 10 \mathrm{sec}^{-1}$ for the dense phase. When these values are introduced into Eqs. (15) and (13) of ref. 14 , one has $\varphi \approx 25 \sim 38$ and $\xi \approx 0.95 \sim$ 0.73 for $U_{f}=10 \mathrm{~cm} / \mathrm{sec}$, and $\varphi \approx 52 \sim 63$ and $\xi \approx$ $0.98 \sim 0.83$ for $U_{f}=40 \mathrm{~cm} / \mathrm{sec}$. Thus, the VUE modification is well applicable to Lewis' reaction data, since $\varphi \gg 10$ and $\xi$ shows little influence on $k_{o r}$ due to $k_{o b}$ being the controlling factor for it.

\section{Test for Data by Lewis, Gilliland and Glass ${ }^{12)}$}

\section{1 Fraction of catalyst, " $a$ "}

As defined in ref. 14, $\mu^{*}$, an apparent volume fraction of catalyst in the bubble phase is related, through Eq. (2), with the fraction of catalyst in the bubble phase, $a$, obtained experimentally by Lewis ${ }^{12}$. On the other hand, the actual fraction of catalyst in the bubble phase, $\mu$ may usually be negligible in comparison with $\mu^{*}$ for fluid beds without internals, because $\mu^{*}$ calculated by the use of Eq. (2) and the experimentally obtained " $a$ " amounts to $0.44 \sim 0.58$ for $U_{f}=10 \sim 40 \mathrm{~cm} / \mathrm{sec}$ and is about two orders greater than experimental $\mu$-values. Under this circumstance, Eq. (17) of ref. 14 is reduced to

$$
\mu^{*} \varepsilon_{b}=\int_{1}^{Z_{t}} \varepsilon_{e} d Z
$$

By introducting Eq. (4) into Eq. (2) and then utilizing a formal relation for the dense phase that $\varepsilon_{e}=\int_{0}^{1} \varepsilon_{e} d Z$, one has

$$
a=\int_{1}^{Z_{t}} \varepsilon_{e} d Z / \int_{0}^{Z_{t}} \varepsilon_{e} d Z=\frac{H_{f}-H_{t}}{H_{o}-H_{t}}
$$

where $H_{o}, H_{f}$, and $H_{t}$ are the manometer readings at $z=0, L_{f}$ and $L_{t}$ respectively (ref. to Fig. 2 of ref. 14). The term " $a$ " has been evaluated by utilizing Eq. (5) and Figs. 2 and 3 of ref. 14 for the bed with $d_{p}=128 \mu$ and $L_{q}=20 \sim 60 \mathrm{~cm}$. Figure 1a compares " $a$ " thus evaluated with that given by Lewis et $a .^{12)}$ Unexpectedly good agreement is observed between these two. Also, different quiescent bed heights give about the same " $a$ " in accordance with Lewis. This consistency may perhaps be somewhat fortuitous, since there is ambiguity ${ }^{14)}$ in defining the location of $L_{f}$. In spite of this, the successive contact model is seen to provide a reasonable behaviour of the bed in accordance with the reaction performance.

Figure 1b illustrates " $a$ " determined for $85 \mu$ FCC catalyst (Fig. 4 of ref. 14) and that given by Lewis for $80 \mu$ alumina catalyst impregnated with nickel. Agreement is moderate, perhaps due to difference in density between the two catalysts.

In applying Figs. $2 \sim 4$ of ref. 14 to obtain " $a$ ", it has been tacitly assumed that distribution of particles in the dilute phase fluidized by air is equally applicable to the case of ethylene fluidization at the reaction temperature. The terminal velocity of the catalyst particle in air has been estimated as being by about $25 \%$ less than that in ethylene, both being in the Stokes regime. Movement of particles in the dilute phase is essentially in mutually hindered motion, so that the influence of gas properties is assumed to tend to diminish.

\section{2 Mass transfer term, $F$}

Since the term " $a$ " has shown good agreement between the two models, the term $F$ is necessarily equivalent in the two for $122 \mu$ dia. particles. As shown in Fig. 2, $k_{o b} a_{b} / \varepsilon_{b}$ has been obtained as a function of $U_{f}$, by introducing experimental data ${ }^{12)}$ for 
$F$ into Eq. (3), where $\varepsilon_{b}$ has been determined* from Figs. 2 and 3 of ref. 14 . Two curves of $L_{1}$ and $L_{2}$ are thus calculated respectively for 122 and $80 \mu$ dia. particles.

To compute the mass transfer term analytically, a mean bubble diameter $d_{b}$ is needed for each $U_{f}$. This size has been given by Morooka ${ }^{17)}$ for fluid beds with 79 and $195 \mathrm{~mm}$ dia. which fluidize 59 and $80 \mu$ dia. FCC catalyst particles. For a $50 \mathrm{~mm}$ dia. bed, Furusaki $^{5)}$ has measured the number mean bubble size $\left(1.7 \sim 4.6 \mathrm{~cm}\right.$ for $\left.U_{f}=10 \sim 40 \mathrm{~cm} / \mathrm{sec}\right)$ for $\mathrm{FCC}$ catalyst of similar size as above. Morooka's number mean bubble size is in $2.0 \sim 4.0 \mathrm{~cm}$ for $U_{f}=10 \sim 40 \mathrm{~cm} /$ $\mathrm{sec}$, and is about the same as that given by Furusaki. Morooka $^{17)}$ also gives the volume-surface mean bubble size for the same data as above, and this will be taken as the mean bubble diameter $d_{b}$ for the following computation $\left(d_{b}=4.0 \sim 8.0 \mathrm{~cm}\right.$ for $U_{f}=10 \sim 40 \mathrm{~cm} /$ sec and $D_{T}=79$ and $195 \mathrm{~mm}$ ).

Assuming freely rising bubbles through the dense phase, $k_{o b} a_{b} / \varepsilon_{b}$ was calculated (refer to Appendix), and is shown in Fig. 2. As has been discussed in Appendix (Section A.2), it seems reasonable to take into consideration the influence of the Hatta number $\beta_{r}$ on $k_{o b}$. This influence is also observed from the predicted curves of $\mathrm{A}$ and $\mathrm{B}$ as shown in Fig. 2, where larger $K\left(=\lambda k_{r}\right)$ value gives higher $k_{o b} a_{b} / \varepsilon_{b}$. Due to this, Eq. (A.6) has been utilized to predict the rate of gas exchange during reaction.

In Fig. 2, the cross-hatched curve $\mathrm{A}$ has been determined from Eqs. (A.6) (A.9), and Curve B is from the same equations by utilizing Eq. (A.15) instead of Eq. (A.7). Prediction seems to be about reasonable. Curve A, which has been calculated from the bubble sizes originally determined for 59 and $80 \mu$ dia. FCC particle beds, fits fairly well with the data for $80 \mu$ dia. alumina particle bed. This curve, however, fails to account for the data for $122 \mu$ dia. particle bed. Also, visually it is somewhat difficult to believe that bubble sizes larger than the bed diameter of $5 \mathrm{~cm}$ exist in the bed. Perhaps the effective bubble sizes may be somewhat smaller than those assumed here, and the increase in $k_{o b} a_{b} / \varepsilon_{b}$ due to decrease in the bubble sizes is cancelled as the result of the rising velocity of bubbles being retarded by the wall effect ${ }^{13,21,22)}$. More work is needed to make these phenomena clear.

\section{Test for Data by Gilliland and Knudsen ${ }^{7)}$}

\footnotetext{
* $\varepsilon_{b}=0.12,0.18,0.23,0.27$, and 0.30 for $U_{f}=10,20,30,40$, and $50 \mathrm{~cm} / \mathrm{sec}$ respectively. These $\varepsilon_{b}$ are somewhat lower than those given by Morooka et al. ${ }^{18)}$

** Graphical plotting of $K Q /(K-Q)$ vs. $K^{2} /(K-Q)$, however, gives good straight lines for each constant $U_{f}$ series. Another source of inaccuracy is the assumption of the reaction being isothermal even in the dilute phase.
}

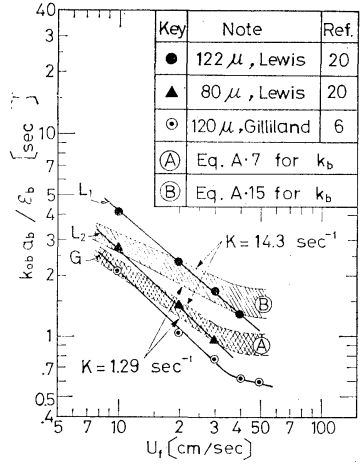

Fig. 2 Comparison of overall mass transfer term $\boldsymbol{k}_{o b} a_{b} / \boldsymbol{\varepsilon}_{b}$, as a function of $U_{f}$ from several sources.

Reaction data have been given by them ${ }^{7)}$ for a 2 -in. diameter fluidized bed reactor, using ethylene hydrogenation on various particle sizes of copperimpregnated fluid cracking catalyst. Their series 52,54 and 56 runs furnish enough information to be analysed further by the "direct contact model" (refer to their Figs. 4 and 5). Two terms of $F$ and " $a$ " are obtained by plotting $K Q /(K-Q)$ vs. $K^{2} /(K-Q)$ in essentially same way as Lewis et al. ${ }^{12)}$

The mass-transfer term $k_{o b} a_{b} / \varepsilon_{b}$ calculated from $F$, which is obtained as above, is plotted as Curve $\mathrm{G}$ in Fig. 2 for $120 \mu$ dia. catalyst particles. The curve is located in about the same order of magnitude as those by Lewis.

The fraction of catalyst " $a$ " is also plotted in Fig. 1a as a function of $U_{f}$. The catalyst activity $K$ converted from Gilliland's intrinsic activity for those runs is $0.83,0.48$ and $0.24 \mathrm{sec}^{-1}$ for series 52,54 and 56 respectively, and is fairly lower than those by Lewis $\left(K=1.29 \sim 14.3 \mathrm{sec}^{-1}\right)$. Due to this, accuracy for determining $F$ and " $a$ " may not** be so good as that in the case of Lewis' data. With this in mind, it is still quite interesting to see that the term " $a$ " is surprisingly greater than those by Lewis.

When " $a$ " calculated as above is converted to $\mu^{*}$ by utilizing Eq. (2), $\mu^{*}$ amounts to 7.6 to 5.5 for $U_{f}=10 \sim 50 \mathrm{~cm} / \mathrm{sec}$. In other words, the volume of catalyst (with an apparent density the same as the emulsion phase) which contacts freely with the bubble gas amounts to 7.6 to 5.5 times the volume of the bubble gas itself. Obviously this is not sound physically, even if the above $\mu^{*}$ are rather overestimated due to lack of accuracy**.

On the other hand, this amount of freely contacting catalyst could possibly be suspended in the dilute phase, since the catalyst had a much less quiescent bed density of $0.33 \mathrm{~g} / \mathrm{cc}$. There is an experimental observation which supports this possibility. Utilizing the fluid bed shown in Fig. 1 of ref. 14, bed density distribution was measured for air-fluidized microspherical alumina particles (MSC-1A pre- 
pared by Mizusawa Kagaku Co.) with $88 \mu$ average diameter. The quiescent bed density was $0.833 \mathrm{~g} / \mathrm{cc}$. $L_{q}$ was changed from 20 to $60 \mathrm{~cm}$, and $U_{f}$ from 20 to $60 \mathrm{~cm} / \mathrm{sec}$. The volume fraction of particles suspended in the dilute phase decreased for MSC-1A particles to about one half of that for FCC catalyst of $85 \mu$ diameter (ref. to Fig. 4 of ref. 14). Thus, at least qualitatively, it is quite probable for the series by Gilliland that a fair amount of catalyst particles is suspended in the dilute phase.

\section{Discussion and Remarks}

The kinetic approach presented here as the "successive contact model" has been formulated in its simplest version for more complicated fluid bed behaviour (Sections 2.2 and 2.3 of ref. 14). In spite of this, the test of the model has not revealed any contradiction with observations (Sections 3 and 4). Quite large $\mu^{*}$ obtained in Section 4 seems to provide support to this approach. Physical quantities introduced into this new model are measurable by independent experimental methods, as have been estimated frequently in Section $2 \sim \mathbf{4}$.

In fluid beds without internals, progress of reaction in the dilute phase has been revealed as having an important role in overall conversion under relatively high gas velocity. The reaction in the dilute phase seems to proceed under relatively low axial dispersion of reacting gas. When the reaction proceeds extensively in the phase, a temperature distribution is built up axially. This distribution has no particular ill effect on the progress of simple irreversible reaction, but may reveal sometimes a drastic effect on the selectivity of successive or simultaneous reaction. The selectivity depends on the relative magnitude of activation energies of each reaction step, and is influenced directly by the temperature distribution.

Hence, when the temperature does not too much influence the selectivity, and the rate of reaction is enough high, progress of reaction in the dilute phase would even be desirable, because good fluid-particle contact is realized in the dilute phase under higher gas velocity.

Fluid beds with internals are frequently utilized for exothermic reactions. Qualitative implication of the internals has been discussed extensively ${ }^{8,26)}$. Lewis $^{12)}$ have shown quantitatively the influence of baffles on the progress of reaction. Overall effect of the internals is, thus, supposed first to increase $k_{o b} a_{b}$ and perhaps $\mu$ (not $\mu^{*}$ ), and secondly to decrease the catalyst content in the dilute phase by reducing both the circulation rate of the dense phase and the ascending velocity of bubbles.

The successive contact model seems to provide a reasonable approach to fluid bed design, by making it feasible to take into consideration the influence of bed design as well as the scheme and rate of a given reaction on the optimum selectivity for overall progress of the reaction.

\section{Conclusion}

1. An apparent fraction of catalyst in the bubble phase, $\mu^{*}$, for catalytic hydrogenation of ethylene by fluid beds is shown to be reasonably well estimated from catalyst distribution in the dilute phase. The new approach provides a sound explanation for an apparent anomaly of $\mu^{*}$ being fairly greater than unity for some published data.

2. Influence of bed design and operation on selectivity of a given reaction is briefly given for fluid beds.

3. The concept of diffusion with simultaneous adsorption and chemical reaction is shown to provide a sound approach for determining the overall coefficient of mass transfer between the bubble void and the emulsion phase during reaction in a fluid bed.

\section{Appendix}

\section{A-1 Prediction of gas exchange coefficient}

Davidson and Harrison ${ }^{31}$ have assumed that there are both convective and diffusional flows which contribute to the gas exchange between the bubble and emulsion phases. Partridge and Rowe ${ }^{20)}$ have developed a boundary layer equation for the transfer from cloud to emulsion phase. Kunii and Levenspiel ${ }^{9,10)}$ have proposed a model for estimating the gas exchange coefficient. They assume that there are two transfer steps, namely the transfer between bubble void and cloud-particle overlap region and that between the cloud-particle overlap region and the emulsion phase. The mass transfer coefficient for the former step is assumed as being given by that of Davidson and Harrison, and for the latter step the Higbie penetration model is supposed to be well applicable. In terms of mass transfer coefficient their equation is given as follows:

$$
1 / k_{o b}=1 / k_{b c}+1 / k_{c e}
$$

where

$$
\begin{aligned}
& k_{b c}=(3 / 4) U_{m f}+0.975 D_{G}^{1 / 2}\left(g / d_{b}\right)^{1 / 4} \\
& k_{c e} \doteqdot(2 / \sqrt{\pi})\left(\varepsilon_{m} f^{2} D_{G} u_{b} / d_{b}\right)^{1 / 2}
\end{aligned}
$$

Recently, Chiba and Kobayashi') have presented the following equation based on the Murray stream function ${ }^{19)}$ :

$$
k_{o b}=\frac{2}{\sqrt{\pi}}\left(\frac{\varepsilon_{m} f^{2} D_{G} u_{b}}{d_{b}}\right)^{1 / 2}\left(1-\frac{1}{\alpha}\right)^{1 / 2}
$$

They further introduce the influence of physical adsorption equilibrium on particles. Their relation is modified as follows in terms of present notation, by using the fact that $\varepsilon_{m f}$ is nearly the same as $\varepsilon_{f e}$ :

$$
\left(k_{o b}\right)_{m_{s} \neq 0} /\left(k_{o b}\right)_{m_{s}=0}=\eta \sqrt{m / \varepsilon_{f e}}
$$

where

$$
\eta=\sqrt{1+\frac{2}{3}\left(1-\frac{\varepsilon_{f e}}{m}\right)\left(\frac{1}{a-1}\right)}
$$

Gas exchange during catalytic reaction between bubbles and the emulsion phase of catalyst particles is essentially a diffusion phenomenon with simultaneous adsorption and chemical reaction in the emulsion phase. Based on this physical 
picture, Miyauchi and Morooka ${ }^{16)}$ have developed the following relation for $k_{o b}$.

$$
1 / k_{o b}=1 / k_{b}+1 / \beta_{r} k_{e}
$$

where

$$
\begin{aligned}
& k_{b}=(2 / \sqrt{\pi}) \sqrt{D_{G} u_{b} / d_{b}} \\
& k_{e}=(2 / \sqrt{\pi}) \sqrt{m D_{e f f} u_{b} / d_{b}}
\end{aligned}
$$

and

$$
\beta_{r}=\beta-\left(\varepsilon_{f e} / m\right) J
$$

The term $\beta$ is the Hatta number* for unsteady gas absorption with chemical reaction as given by Danckwerts ${ }^{2)}$, and is found in standard texts as a function of $m_{H}\left(=\sqrt{k_{r} D_{e f f}} / k_{e}\right) . \quad J$ is the term associated with the gas cloud, and is found from Fig. 3 as a function** of $m_{H}$ and $P e$ (the gas cloud parameter).

Relation between the proposed equations is as follows. Eq. (A.6) is about the same as Eq. (A.1) for smaller $U_{m f}$, when $\beta_{r}=1$ (no reaction) and $m=\varepsilon_{m f}$ (no partition to particles). The latter condition is that Eqs. (A.3) and (A.8) are different in general, due to $m$ being not necessarily equal to $\varepsilon_{m f}$. Bubble void resistance to mass transfer has been assumed as being negligible in Eq. (A.4). This equation is applicable to the case of arbitrary $m$ by utilizing Eq. (A.5). Eq. (A.8) is rewritten in similar form to Eq. (A.5) as follows (note that $m=\varepsilon_{f e}+m_{s} \varepsilon_{s e}$, and $\left.\varepsilon_{f e} \fallingdotseq \varepsilon_{m f}\right)$ :

$$
\left(k_{e}\right)_{m_{s} \neq 0} /\left(k_{e}\right)_{m_{s}=0}=\sqrt{m / \varepsilon_{f e}}
$$

The correction factor $\eta$ to Eq. (A.5) is nearly equal to unity for $\alpha \geq 10$, so that Eq. (A.5) is almost equivalent to Eq. (A.10) for smaller particles. Eq. (A.4) is independent of $m_{H}$ or the rate of chemical reaction.

Recently, Drinkenburg and Rietema ${ }^{4 /}$ have presented a numerical computation of $k_{o b}$ based on the stream functions given by Davidson and Harrison ${ }^{31}$ and by Murray ${ }^{19}$. The bubble void resistance to mass transfer has been neglected. Enhancement of gas transfer rate by diffusion with simultaneous chemical reaction (Fig. 5 of ref. 4) seems to be reasonably well expressed by Eq. (A.9), when the enhancement is expressed as the Hatta number. Enhancement by physical adsorption (Fig. 2 of ref. 4) is also approximated by Eq. (A.5) or Eq. (A.10) for smaller particles.

When the reaction data by Lewis ${ }^{12)}$ have been analysed by utilizing Eq. (A.6), it has turned out that $\beta_{r}$ amounts to 1.45 (free flow bubbles) and higher values than 1.45 (bubbles retarded by the wall effect) for the highest reaction rate constant of $K=$ $\lambda k_{r}=14.3 \mathrm{sec}^{-1}$. Hence it seems reasonable to take into consideration the influence of $\beta_{r}$ on $k_{o b}$. Also, it has been shown experimentally that $k_{b}$ is not necessarily negligible for fluid beds ${ }^{23)}$.

Due to these, Eq. (A.6) has been utilized, in what follows, to predict the rate of gas exchange during reaction.

When $P e \leqslant 10^{-2}$ and $m_{H} \gtrsim 3$, Eq. (A.9) reduces to $\beta_{r} \doteqdot \beta \doteqdot m_{H}$ $\left(=\sqrt{k_{r} D_{e f f}} / k_{e}\right)$. Hence, the following simple relation is obtained for $k_{o b}$ from Eq. (A.6).

$$
\begin{aligned}
& * \beta=\left(m_{H}+\frac{\pi}{8 m_{H}}\right) \operatorname{erf}\left(\frac{2 m_{H}}{\sqrt{\pi}}\right)+\frac{1}{2} \exp \left(-\frac{4 m_{H}{ }^{2}}{\pi}\right) \\
& * * \quad J=\frac{\pi}{4 m_{H}} \operatorname{erf}\left(\frac{2 m_{H}}{\sqrt{\pi}}\right)-\int_{0}^{1} \frac{1}{2 \sqrt{\phi}} \exp \left[-\left(\frac{4 m_{H}{ }^{2}}{\pi} \phi+\frac{P_{e}}{\phi}\right)\right] d \phi
\end{aligned}
$$

where $\phi$ is an integration parameter, and

$$
\begin{aligned}
& (J)_{m_{H}=0}=1-e^{-P e}+\sqrt{\pi P e} \operatorname{erfc} \sqrt{P e} ;(J)_{\left.\left.m_{H}\right\rangle\right\rangle 1}=0, \\
& (J)_{m_{H}=0, P e} \leqslant 0.1=\sqrt{\pi P e}-P e,(\max . \text { error }=-2.6 \%)
\end{aligned}
$$

and

$$
(J)_{P e=0}=0 \text {. }
$$

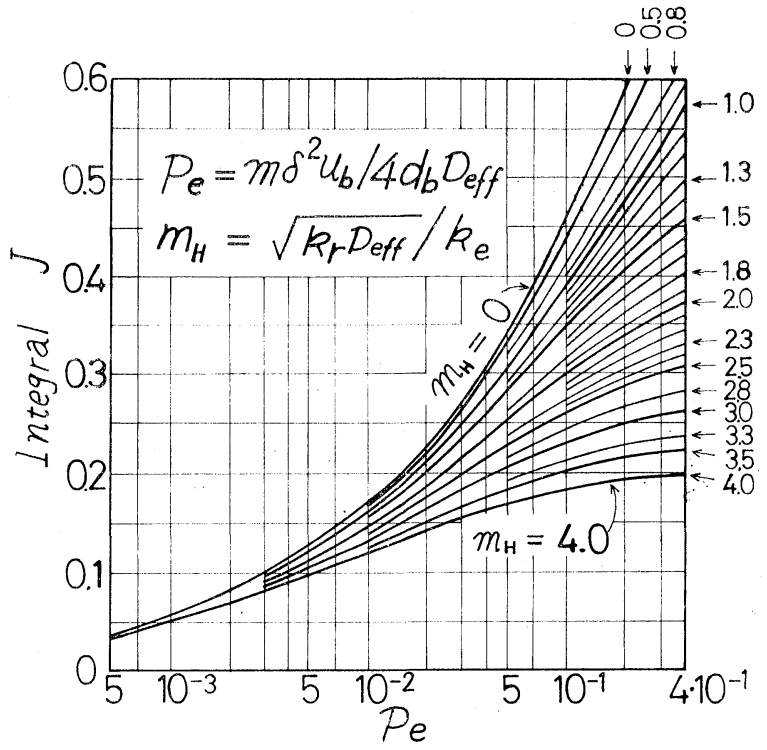

Fig. 3 Integral $J$ as a function of $P e$ and $m_{H}$

$$
1 / k_{o b}=1 / k_{b}+1 / \sqrt{k_{r} D_{\text {eff }}}
$$

$k_{o b}$ is independent of $k_{e}$ under such circumstances.

\section{A-2 Computation of $\boldsymbol{k}_{o b}$ by utilizing Eq. (A.6)}

Physical data necessary for the computation are $D_{G}, m, k_{r}$, $U_{m f}, d_{b}$ and $\varepsilon_{f e}\left(\doteqdot \varepsilon_{m f} \doteqdot 0.50\right)$.

By knowing these basic data, several quantities are then computed from the following relations:

$$
\begin{aligned}
u_{b} & \left.=0.71 \sqrt{g d_{b}} \text { (free flow bubble) }\right)^{3)} \\
\alpha & =u_{b} /\left(U_{m f} / \varepsilon_{m f}\right) \\
\delta & =\frac{d_{b}}{2}\left[\left(\frac{\alpha}{\alpha-1}\right)^{1 / 3}-1\right]: \quad(\text { ref. } 19) \\
D_{e f f} & =\varepsilon_{f e} D_{G} / \chi \doteqdot D_{G} / 3.0 \quad\left(\because \varepsilon_{f e} \doteqdot 0.5, \chi \doteqdot 1.5\right) \\
k_{b} & =\text { Eq. (A.7), } \quad k_{e}=\text { Eq. (A.8) }
\end{aligned}
$$

Since the particle Péclet number $d_{p} U_{m f} / D_{G}$ for the emulsion phase in fluid beds is usually below 0.1 (refer to the footnote of Section 3.2 of ref. 14), the effective diffusivity may be well expressed by the above equation ${ }^{6,15)}$, where the tortuosity factor is taken according to the value given by Hoogschagen ${ }^{15}$. $P e$ and $m_{H}$ are then obtained as follows:

$$
\begin{aligned}
& P e=m \delta^{2} u_{b} / 4 d_{b} D_{e f f} \\
& m_{H}=\sqrt{k_{r} D_{e f f}} / k_{e}
\end{aligned}
$$

By knowing $m_{H}, \beta$ in Eq. (A.9) is determined from the Hatta number for gas absorption with chemical reaction. Also, $J$ is obtained from Fig. 3 for most fluid-bed operations. Hence $\beta_{r}$ is calculated from Eq. (A.9), and $k_{o b}$ from Eq. (A.6).

The computation procedures are considerably simplified when $P e \leqslant 0.005 . \quad k_{o b}$ is simply given by

$$
1 / k_{o b}=1 / k_{b}+1 / \beta k_{e}
$$

According to Morooka ${ }^{17)}$, the volume-surface mean diameter of bubbles, $d_{b}$, is approximately given as $4.0,5.5,7.5,8.0$ and $8.0 \mathrm{~cm}$ for $U_{f}=10,20,30,40$ and $50 \mathrm{~cm} / \mathrm{sec}$, respectively.

Assuming that these bubble diameters are also applicable to $122 \mu$ dia. FCC catalyst and $80 \mu$ dia. alumina catalyst, both utilized by Lewis ${ }^{12)}, k_{o b}$ are calculated by Eq. (A.6), where $m=1$ and $D_{G}=0.904 \mathrm{~cm}^{2} / \mathrm{sec}$ for $\mathrm{C}_{2} \mathrm{H}_{4}-\mathrm{H}_{2}$ system at $113^{\circ} \mathrm{C}$ and 1 atm. Also, $U_{m f}=0.73 \mathrm{~cm} / \mathrm{sec}, \varepsilon_{f c}=0.492$ (average), and $k_{r}=K / 1.09$ with $K=1.29 \sim 14.3 \mathrm{sec}^{-1}$ according to Lewis ${ }^{12)}$. $k_{o b} a_{b} / \varepsilon_{b}\left(=6 k_{o b} / d_{b}\right)$ thus obtained is shown in Fig. 2 as the cross-hatched curve A.

Experimentally measured bubble-phase coefficient of mass 
transfer has been given by Yamazaki ${ }^{23)}$ as

$$
k_{b} a_{b} / \varepsilon_{b}\left(=6 k_{b} / d_{b}\right)=3.66 U_{f^{1 / 3}}(0.66 / S c)^{1 / 2}
$$

for FCC catalyst particles of $59 \mu$ dia. fluidized by air in 79 and $195 \mathrm{~mm}$ dia. columms. By using this $k_{b}$ (instead of Eq. (A.7)) for Eq. (A.6), $k_{o b} a_{b} / \varepsilon_{b}$ is also calculated and illustrated as Curve B in Fig. 2, where $\mathrm{Sc}=0.167$ for $\mathrm{C}_{2} \mathrm{H}_{4}-\mathrm{H}_{2}$ system.

\section{Acknowledgment}

The author wishes to express his appreciation to Dr. Shigeharu Morooka (Kyushu Univ.) for his providing numerical values for Fig. 3.

\section{Nomenclature}

(See Ref. 14 for additional nomenclature)

$$
\begin{array}{llr}
D_{T} & =\text { bed diameter } & {[\mathrm{cm}]} \\
D_{e f f} & =\text { effective diffusivity } & {\left[\mathrm{cm}^{2} / \mathrm{sec}\right]} \\
F & =\text { ref. to Eq. (3) } & {[1 / \mathrm{sec}]} \\
H & =\text { manometer reading } & {[\mathrm{cm}]} \\
\bar{H}_{0}, H_{f}, H_{t} & =H \text { at } z=0, L_{f} \text { and } L_{t} \text { respectively } & {[\mathrm{cm}]} \\
J & =\text { refer to Eq. (A.12) } & {[-]} \\
K & =\text { catalyst activity, Eq. (1) } & {[1 / \mathrm{sec}]} \\
P e & =m \delta^{2} u_{b} / 4 d_{b} D_{\text {eff }} & {[-]} \\
Q & =\text { specific converting power } & {[1 / \mathrm{sec}]} \\
S c & =\text { Schmidt number } & {[-]} \\
a & =\text { refer to Eq. }(2) & {[-]} \\
d_{b} & =\text { bubble diameter } & {[\mathrm{cm}]}
\end{array}
$$$$
k_{b}, k_{e} \quad=\text { film coefficients for bubble phase and }
$$
emulsion phase, respectively $\quad[\mathrm{cm} / \mathrm{sec}]$

$k_{b c}, k_{c e} \quad=$ refer to Eqs. (A.2) and (A.3) [cm/sec]

$m_{H} \quad=\sqrt{k_{r} D_{\text {eff }}} / k_{e} \quad[-]$

$u_{b} \quad=$ mean interstitial velocity of bubbles relative to the emulsion phase $\quad[\mathrm{cm} / \mathrm{sec}]$

$\alpha \quad=$ ratio of $u_{b}$ to $U_{m f} / \varepsilon_{m f} \quad[-]$

$\beta \quad=$ Hatta number for gas absorption with reaction, Eq. (A.11) [-]

$\beta_{r} \quad=$ refer to Eq. (A.9) [-]

$\delta \quad=$ thickness of cloud-particle overlap region [cm]

$\varepsilon_{m f} \quad=$ void fraction at $U_{m f} \quad$ [-]

$\eta \quad=$ refer to Eq. (A.5) [-] $\begin{array}{rll}\lambda & =\varepsilon_{s q} / \varepsilon_{s e} \doteqdot 1.09 & {[-]} \\ \chi & =\text { tortuosity factor } \fallingdotseq 1.5 & {[-]}\end{array}$

\section{Literature Cited}

1) Chiba, T. and H. Kobayashi: Chem. Eng. Sci., 25, 1375 (1970)

2) Danckwerts, P. V.: Trans. Farad. Soc., 46, 300 (1952)

3) Davidson, J. F. and D. Harrison: "Fluidized Particles", Cambridge Univ. Press, London (1963)

4) Drinkenburg, A. A. H. and K. Rietema: Chem. Eng. Sci., 27, 1765 (1972)

5) Furusaki, S.: AIChE J., 19, 1009 (197)

6) Gunn, D. J.: Trans. Inst. Chem. Engrs., 47, T351 (1969)

7) Gilliland, E. R. and C. W. Knudsen: Chem. Eng. Prog., Symp. Ser., 67, No. 116, 168 (1971)

8) Ikeda, Y.: Kagaku Kögaku, 29, 57 (1965); 34, 1013 (1970)

9) Kunii, D. and O. Levenspiel: Ind. Eng. Chem., Fund., 7, 446 (1968)

10) Kunii, D. and O. Levenspiel: "Fluidization Engineering", Wiley, N.Y. (1969)

11) Lewis, W. K., E. R. Gilliland and H. Girouard: Chem. Eng. Prog., Symp. Ser., 58, No. 38, 87 (1962)

12) Lewis, W. K., E. R. Gilliland and W. Glass: AIChE J., 5, 419 (1959)

13) Matsen, J. M.: Chem. Eng. Prog., Symp. Ser., 66, No. 101, 47 (1970)

14) Miyauchi, T.: J. Chem. Eng. Japan, 7, 201 (1974)

15) Miyauchi, T.: ibid., 4, 238 (1971)

16) Miyauchi, T. and S. Morooka: Kagaku Kögaku, 33, 369, 880 (1969); Intern. Chem. Eng., 9, 713 (1969)

17) Morooka, S., K. Tajima and T. Miyauchi: Kagaku Kōgaku 35, 680 (1971); Intern. Chem. Eng., 11, 168 (1972)

18) Morooka, S., Y. Kato and T. Miyauchi: J. Chem. Eng. Japan, 5, 161 (1972)

19) Murray, J. D.: J. Fluid Mech., 22, 57 (1965)

20) Partridge, B. A. and P.N. Rowe: Trans. Inst. Chem. Engrs., 44, 355 (1966)

21) Squires, A. M.: Chem. Eng. Prog., 58, No. 4, 66 (1962)

22) Strom, J. R. and R. C. Kintner: AIChE J., 4, 153 (1958)

23) Yamazaki, M. and T. Miyauchi: J. Chem. Eng. Japan, 4, 324 (1971) 